\title{
Syringoid eccrine carcinoma of the vulva: differential diagnosis, treatment and follow-up.
}

\author{
Grazia Carrara ${ }^{\mathrm{a}}$, Giuseppe Scibiliaa ${ }^{\mathrm{a}}$, Andrea Benedetto Di Stefano ${ }^{\mathrm{a}}$, Basilio Pecorino ${ }^{\mathrm{a}}$,
}

Maria Cristina Teodoro ${ }^{\mathrm{a}}$, Giuseppa Scandurra ${ }^{\mathrm{b}}$, Maria Grazia Tranchinac and Paolo Scolloa

a'Gynecology and Obstetrics Unit, ${ }^{\mathrm{b} M e d i c a l}$ Oncology Unit, ${ }^{\mathrm{c} A n a t o m i c a l ~ P a t h o l o g y ~ U n i t ~}$

Azienda ospedaliera per l'emergenza, Ospedale Cannizzaro, Catania, Italy

Introduction. Syringoid eccrine carcinoma (SEC) is an extremely rare malignant adnexal tumor with eccrine differentiation first described by Freeman and Wilkemann in 1969 as eccrine epithelioma. Its name originates from histological pattern which resembles that one of a syringoma by showing ductal, cystic and comma-like epithelial components in a fibrocollagenous matrix. Fewer than 50 cases have been reported under the name of SEC and its synonysms. Due to limited availability of literature, there is a lack of a universally accepted staging system and the diagnosis and management of these tumors are difficult.

Purpose. The aim of this study is to report the extremely rare case of a syringoid eccrine carcinoma of the vulva occurred in a 33 years old woman who referred to our Gynecology Unit on January 2017, speculating on differential diagnosis, prognostic criteria, management and follow up.

Methods. Accepted criteria on haematoxylin and eosin sections were used to make the diagnosis. Two pathologists evaluated formalin-fixed tissue sections independently, reaching the same conclusions. SEC is a dermal lesion without epidermal connection. Epithelial cells are usually organized in strands with lumen formation, which is called syringomalike 'tadpole' morphology. Basaloid cells have uniform hyperchromatic nuclei. There usually is an infiltrative growth pattern with deep invasion and extension into the subcutaneous tissue or skeletal muscle, and a common perineural and lymphovascular invasion. Mitotic activity is variable.

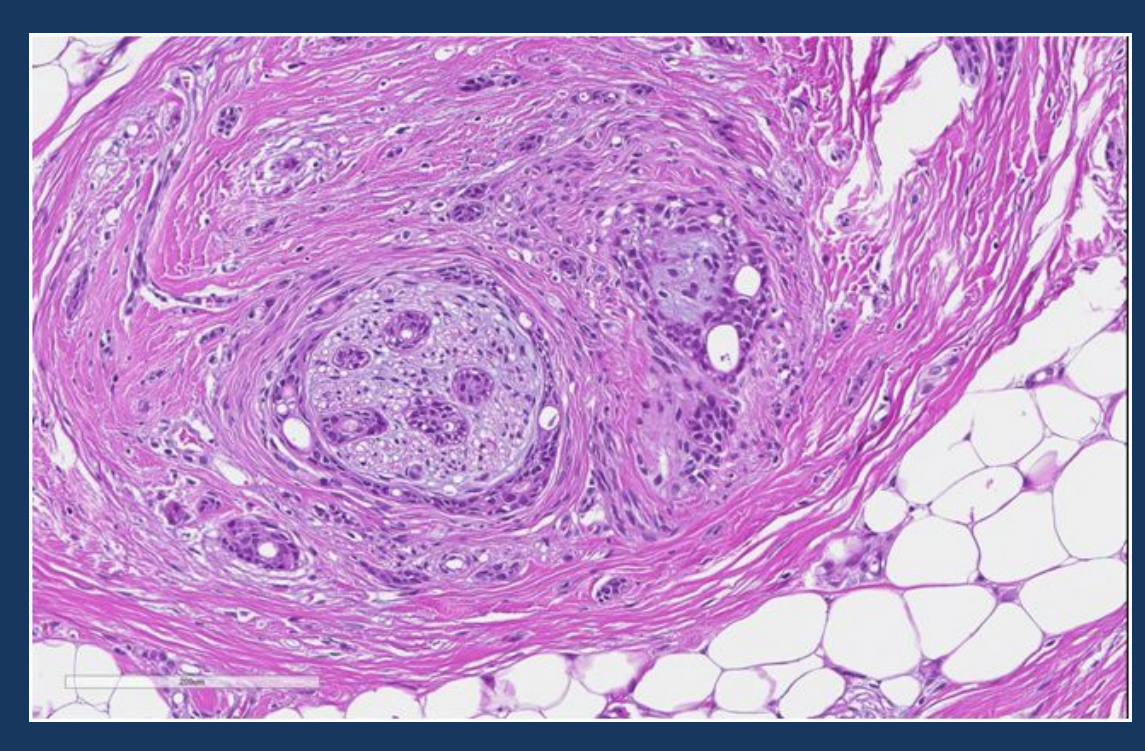

Differential diagnosis. The differential diagnosis of SEC includes primary cutaneous adenoid cystic carcinoma (PCACC), microcystic adnexal carcinoma, infundibular carcinoma, adenocarcinoma with skin metastases, sclerosing basal cell carcinoma (BCC), desmoplastic trichoepithelioma and syringoma.

PCACC is a rare, slow-growing tumor that consists of cords of basaloid cells, characteristic sieve-like cribriform growth and mucin production, not usually present in SEC. The tumor cells are typically small, cuboidal and monomorphic.

Microcystic adnexal carcinomas show both eccrine and follicular differentiation with basaloid cells forming keratin-filled cysts, absent in SEC. Common features of infundibular carcinoma are hair follicular infundibulum attachment, sheets of basaloid cells organized in small nests superficially, pseudoglandular pattern in deep and squamous differentiation; desmoplastic stroma is the only similarity with SEC. Metastatic adenocarcinoma of the skin usually presents in patients with a clinical history of preexisting disease.

Carcinoma of the breast, lung, kidney, colon, stomach and ovaries can be differentiated from SEC usually through morphology and immunohistochemistry. Compared to primary eccrine carcinomas, metastatic adenocarcimonas are generally high grade lesions with remarkable atypia.

Sclerosing BCC shows infiltrative basaloid cells organized in palisading arrangement. SEC never demonstrates the palisading arrangement seen in BCC and other hair follicular-derived carcinomas. It is an epidermal tumor with prominent scleroting stroma that does not have lumen formation. There can be 'tadpole' shaped ductal structures, but they are uncommon. Other variants of BCC help in diagnosis since they often coexist with the sclerosing variant, such us nodular, micronodular or superficial multicentric tumors. Cellular atypia is common.

Desmoplastic tricoepithelioma is tipically a cutaneous lesion with a central dell. Microscopic features are 'tadpole' shaped ductal structures in a sclerotic stroma, clefts between collagen fibres, calcifications and keratinous cysts.

Syringomas are the benign counterparts of SEC. They most commonly present as multiple small flesh-colored papules. On histology, they are superficial dermal lesions made of small ducts lined by benign cuboidal cells forming 'tadpole'-like structures within a fibrocollagenous matrix without perineural and lymphovascular involvement. Cellularity, anaplasia and deep invasiveness are pivotal element in differential diagnosis between syringoma and SEC.

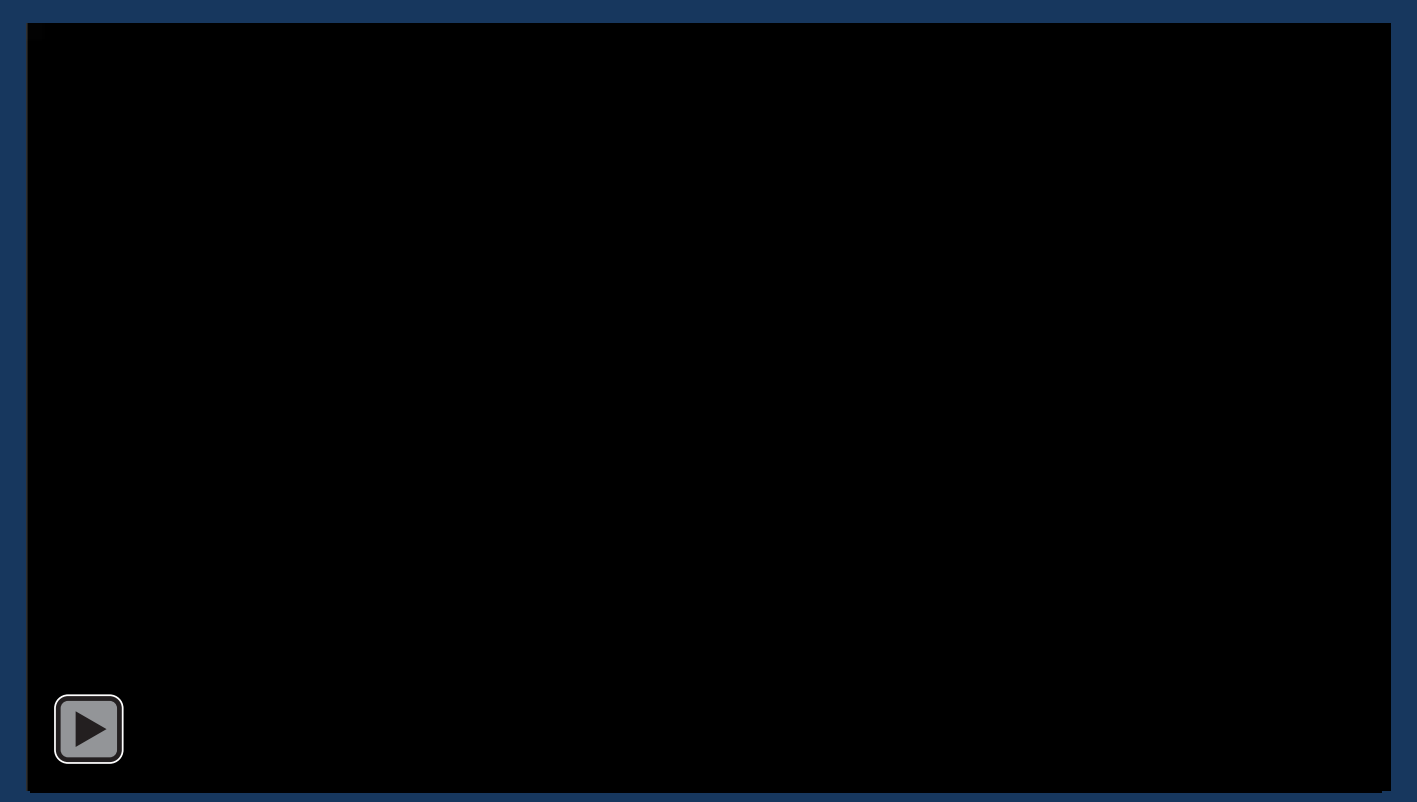

Results. The patient underwent a right anterior hemivulvectomy with minor labrum preservation, without complications and next day discharge. Resection margins were tumor-free, obtaining oncologic radicality (FIGO stage: IB, Nx). Since SEC has commonly a local recurrence disposition and a potential for distant metastasis, the patient underwent a clinical gynecological evaluation and ultrasonographic bilateral inguinal lymph nodes examination in June 2017, which were found to be negative. The following control in 3 months was negative, too.

Discussion. Despite the low number of cases described in literature, it is unanimously acknowledged that the recommended treatment of all subtypes of sweat gland carcinomas is wide surgical excision associated with regional lymph node dissection in case of clinically positive nodes because of SEC potential for destructive local tissue infiltration and regional as well as distant metastasis. On one hand local reccurrences of these tumors may be massive; on the other, metastases rarely occur, yet they are not impossible.

In literature, there are reports indicating both single-agent and combination chemotherapy have been used infrequently and, at best, have shown only a temporary benefit. Sweat gland carcinomas are commonly considered radio resistant. Prognostic factors are difficult to identify because of the small number of reported cases. Size, histological type, lymph node involvement above all and distant metastases are those considered more relevant. Several features are associated with a poor prognosis: tumor depth $>7 \mathrm{~mm}$, more than 14 mitotic figures on high powered magnification, infiltrative margins; an acute change to a rapid growing lesion could be suggestive for a transition to malignancy.

Survival rates are substantially unknown due to the lack of data. For what concerns apocrine adenocarcinomas, patients without lymph nodes involvement have a 56\% 10-year disease-free survival rate, which decrease to $9 \%$ if nodes are involved.

Conclusion. SEC should be taken into consideration in vulvar cancer differential diagnosis. A frequent follow up is essential to detect early recurrence or distant metastases. 